\title{
Reinforcement Learning for Orientation Estimation Using Inertial Sensors with Performance Guarantee
}

\author{
Liang $\mathrm{Hu}^{1 *}$, Yujie Tang ${ }^{2 *}$, Zhipeng $\mathrm{Zhou}^{2}$ and Wei $\mathrm{Pan}^{2}$
}

\begin{abstract}
This paper presents a deep reinforcement learning (DRL) algorithm for orientation estimation using inertial sensors combined with magnetometer. The Lyapunov's method in control theory is employed to prove the convergence of orientation estimation errors. Based on the theoretical results, the estimator gains and a Lyapunov function are parametrised by deep neural networks and learned from samples. The DRL estimator is compared with three well-known orientation estimation methods on both numerical simulations and real dataset collected from commercially available sensors. The results show that the proposed algorithm is superior for arbitrary estimation initialisation and can adapt to very large angular velocities for which other algorithms can be hardly applicable. To the best of our knowledge, this is the first DRL-based orientation estimation method with estimation error boundedness guarantee.
\end{abstract}

\section{INTRODUCTION}

Orientation estimation is important in robotics, navigation, control, human motion analysis [1], [2], [3]. Recently, orientation estimation has been greatly advanced by the development of accurate sensors. Multiple sensors are usually combined to estimate the orientation, i.e., sensor fusion. Depending on the availability of sensors and applications, various sensor fusion techniques have been proposed, e.g., the inertial measurement units (IMU) and magnetometer [4], [5], [6], the magnetometer and camera [7], and the IMU and visual sensor [8], [9], etc. In this paper, we focus on orientation estimate using the inertial sensors and magnetometer.

The estimation algorithms can be summarised into three categories: (1) Bayesian estimation, (2) optimisation and (3) deep learning. In Bayesian estimation, the well-known extended Kalman filter (EKF) and the unscented Kalman filter (UKF), were used to estimate the orientation [4], [5], [10]. The key idea is to approximate the orientation states by a Gaussian distribution based on the linearisation technique and the deterministic sampling technique, respectively. Furthermore, the complementary filter was developed based on the EKF, which exploits the complementary characteristics of gyroscopes and that of accelerometer and magnetometer at different time scales [6]. In optimisation, the orientation estimation is obtained based on gradient-based optimisation algorithms [11], [12]. Until recently, the deep learning was introduced to estimate the orientation [13], in which a deep neural network is trained to mimic the noise distribution of gyroscopes

\footnotetext{
*Equal Contribution.

${ }^{1} \mathrm{~L}$. Hu is with the School of Computer Science and Electronic Engineering, University of Essex, UK.

${ }^{2}$ Y. Tang, Z. Zhou and W. Pan are with the Department of Cognitive Robotics, Delft University of Technology, Netherlands. For Correspondence: wei.panetudelft.nl.
}

such that accurate orientation estimates can be obtained by open-loop integration of the noise-free gyro measurements. These algorithms showed superior estimation performance empirically. However, the performance can not be theoretically guaranteed, i.e, the orientation estimate error never diverge. In this paper, we will employ Lyapunov's method in control theory to prove the estimation error boundedness guarantee using samples. Based on the theoretical result, we will develop a DRL-based algorithm to learn the estimator from samples.

Reinforcement learning (RL) was first applied for state estimation in [14]. Motivated by this work, we plan to develop a RL algorithm to learn the estimator gain using samples while the orientation estimator keeps the EKF structure. More specifically, the estimator gain will be approximated by a deep neural network (DNN) as a function of the sequence of estimate errors. Different from other popular RL algorithms [15], [16], [17], the value function will be treated as a Lyapunov function to guarantee the estimation performance. Lyapunov's method has been widely used as a basic tool for stability analysis in control theory [18]. To analyse the stability, the key is to find a scalar "energylike" Lyapunov function for the considered system such that the derivative/difference of Lyapunov function along the state trajectory is semi-negative definite. Nonetheless, the construction/learning of the Lyapunov function in modelfree reinforcement learning is not trivial. [19] proposes a straightforward approach to construct the Lyapunov functions for nonlinear systems using DNNs. Recently, the asymptotic stability in model-free RL is given for robotic control tasks in [20]. Inspired by the works [19], [20], we will also parametrise the Lyapunov function as a DNN and learn the parameters from samples. Thereafter, a new DRL algorithm based on soft actor-critic algorithm [17] that incorporates the Lyapunov boundedness condition in the objective function to be optimised is proposed. By using the learned estimator gain, the estimate error of the orientation estimator is guaranteed to be bounded all the time.

In summary, we combine Lyapunov's method and DRL to design a state estimator with estimation error boundedness guarantee for orientation estimation. The main contribution of this paper is threefold:

1) To the best of our knowledge, this is the first DRL-based orientation estimation method using inertial sensors combined with magnetometer;

2) Estimation error boundedness guarantee is proved using Lyapunov's method in control theory;

3) The proposed algorithm is superior for arbitrary estimation initialisation and can adapt to very large angular 
velocities for which other algorithms, such as the EKF, UKF and complementary filter algorithms, can be hardly applicable.

The rest of the paper is organised as follows. In Section [II. the orientation estimation problem is formulated followed by the preliminaries of Markov decision process. In Section [III] the theoretical result on estimation error boundedness guarantee is proved. In Section IV, a DRL algorithm based on Soft Actor Critic (SAC) combined with theoretical results is proposed to learn the estimator gain. In Section V our method is compared with the EKF, UKF and complementary filter algorithms in simulations and experiments. Conclusion is given in Section VI

\section{PROBlem Formulation}

In this paper, we use the inertial sensors (3D accelerometers and $3 \mathrm{D}$ gyroscopes) combined with the magnetometer to estimate the orientation. As in [3], the system dynamics is standard and our goal is to design the estimator gain. Different from the classic nonlinear filtering techniques based on linearisation, we will show that the estimator gain computation can be formulated as a RL problem.

\section{A. System dynamics and state estimator}

The orientation dynamics is typically given as:

$$
q_{t+1}^{\mathrm{nb}}=q_{t}^{\mathrm{nb}} \odot \exp _{q}\left(\frac{T}{2}\left(y_{\omega, t}-e_{\omega, t}\right)\right),
$$

where $q_{t}^{\mathrm{nb}} \in \mathbb{R}^{4}$ is the unit quaternion for the orientation of the body frame with respect to the navigation frame at time instant $t, T$ is the sampling time, and $y_{\omega, t}$ is the gyroscope measurement. The noise of the gyroscope is a zero-mean Gaussian noise $e_{\omega, t} \sim \mathcal{N}\left(0, \Sigma_{\omega}\right)$ where $\Sigma_{\omega}$ is the covariance of the noise.

Assuming that the linear acceleration is approximately zero, the measurement equations are given as follows:

$$
\begin{aligned}
y_{\mathrm{a}, t} & =-R_{t}^{\mathrm{bn}} g^{\mathrm{n}}+e_{\mathrm{a}, t}, \\
y_{\mathrm{m}, t} & =R_{t}^{\mathrm{bn}} m^{\mathrm{n}}+e_{\mathrm{m}, t},
\end{aligned}
$$

where $y_{\mathrm{a}, t}, y_{\mathrm{m}, t} \in \mathbb{R}^{3}$ are accelerometer and magnetometer measurements at time instant $t$, respectively, $R_{t}^{\mathrm{bn}}$ is the rotation matrix from the navigation frame to the body frame at time instant $t, g^{\mathrm{n}}, m^{\mathrm{n}}$ denote the local earth gravity vector and the local earth magnetic field vector, respectively. The noises $e_{\mathrm{a}, t} \sim \mathcal{N}\left(0, \Sigma_{\mathrm{a}}\right)$, and $e_{\mathrm{m}, t} \sim \mathcal{N}\left(0, \Sigma_{\mathrm{m}}\right)$ with $\Sigma_{m}=\sigma_{m}^{2} \mathcal{I}_{3}$ and $\Sigma_{\mathrm{a}}=\sigma_{\mathrm{a}}^{2} \mathcal{I}_{3}$.

To estimate $q_{t+1}^{\mathrm{nb}}$, the following estimator in terms of the orientation deviation is often proposed [3], [21]:

$$
\begin{aligned}
\hat{q}_{t+1 \mid t}^{\mathrm{nb}} & =\hat{q}_{t \mid t}^{\mathrm{nb}} \odot \exp _{q}\left(\frac{T}{2} y_{\omega, t}\right), \\
\hat{\eta}_{t+1} & =K_{t+1}\left(y_{t+1}-\hat{y}_{t+1 \mid t}\right), \\
\hat{q}_{t+1 \mid t+1}^{\mathrm{nb}} & =\exp _{q}\left(\hat{\eta}_{t+1}\right) \odot \hat{q}_{t+1 \mid t}^{\mathrm{nb}}
\end{aligned}
$$

with

$$
y_{t}=\left(\begin{array}{c}
y_{\mathrm{a}, t} \\
y_{\mathrm{m}, t}
\end{array}\right), \quad \hat{y}_{t+1 \mid t}=\left(\begin{array}{c}
-R\left\{\hat{q}_{t \mid t}^{\mathrm{nb}} \odot \exp _{q}\left(\frac{T}{2} y_{\omega, t}\right)\right\}^{\top} g^{n} \\
R\left\{\hat{q}_{t \mid t}^{\mathrm{nb}} \odot \exp _{q}\left(\frac{T}{2} y_{\omega, t}\right)\right\}^{\top} m^{n}
\end{array}\right),
$$

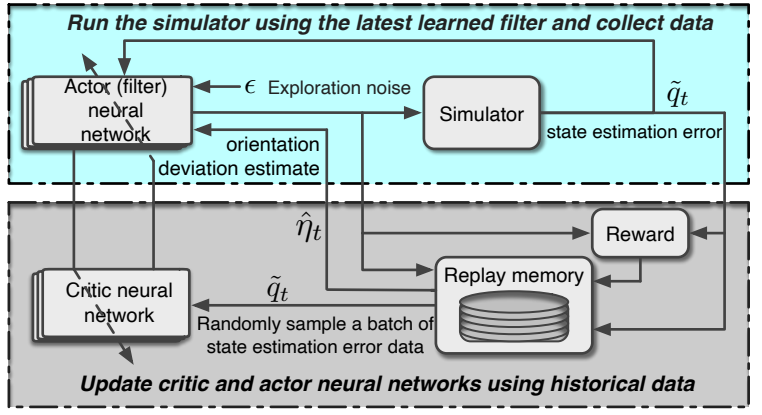

Fig. 1. Offline RL training process of orientation state estimator

where $\hat{q}_{t+1 \mid t}^{\mathrm{nb}}$ is the linearisation point parametrised in terms of quaternions, $\hat{\eta}_{t+1}^{\mathrm{n}}$ is the state estimate of the orientation deviation, and $R\{\}$ denotes the matrix formula of translation from quaternion to rotation. The goal is to obtain $K_{t+1}$, i.e., the estimator gain at time instant $t+1$, which will be described in detail in the next subsection.

Define the orientation error

$$
\tilde{q}_{t} \triangleq q_{t}^{\mathrm{nb}} \odot\left(\hat{q}_{t \mid t}^{\mathrm{nb}}\right)^{\mathrm{c}},
$$

or equivalently $q_{t}^{\mathrm{nb}}=\tilde{q}_{t} \odot \hat{q}_{t \mid t}^{\mathrm{nb}}$. From (1), (2) and (3), we have

$$
\begin{aligned}
& \tilde{q}_{t+1}=q_{t+1}^{\mathrm{nb}} \odot\left(\hat{q}_{t+1 \mid t+1}^{\mathrm{nb}}\right)^{\mathrm{c}} \\
= & \left(\left(\tilde{q}_{t} \odot \hat{q}_{t \mid t}^{\mathrm{nb}}\right) \odot \exp _{q}\left(\frac{T}{2}\left(y_{\omega, t}-e_{\omega, t}\right)\right)\right) \odot \\
& \left(\left(\exp _{q}\left(\frac{1}{2} K_{t+1}\left(y_{t+1}-\hat{y}_{t+1 \mid t}\right)\right) \odot \hat{q}_{t \mid t}^{\mathrm{nb}} \odot \exp _{q}\left(\frac{T}{2} y_{\omega, t}\right)\right)\right)^{\mathrm{c}}
\end{aligned}
$$

Furthermore, to escape the unit determinant condition of the quaternion representation of rotation, the logarithm map of the quaternion is used [22]:

$$
\left[\eta_{t+1}\right]_{\times}=\log \left(\tilde{q}_{t+1}\right)
$$

where $\eta_{t+1}$ is the orientation deviation and the skew operator $[\cdot]_{\times}$produces the cross-product matrix.

\section{B. Estimate Error Dynamics as Markov Decision Process}

By combining (5) and (6), the dynamics of estimate error can be viewed as a Markov decision process (MDP) which is defined as a tuple $\langle\mathcal{S}, \mathcal{A}, \mathcal{P}, \mathcal{C}, \gamma\rangle$ :

$$
\tilde{q}_{t+1} \sim \mathcal{P}\left(\tilde{q}_{t+1} \mid \tilde{q}_{t}, K_{t+1}\right), \forall t \in \mathbb{Z}_{+},
$$

where the estimate error $\tilde{q}_{t} \in \mathcal{S}$ is the state, the estimator gain $K_{t+1} \in \mathcal{A}$ is the action sampled from a stochastic policy. Considering that the ground truth $q_{t}$ is known during training phase, the mapping between $\tilde{q}_{t}$ and $\hat{\eta}_{t}$ is bijective to some extent according to (3c) and (4). For convenience, in our implementation of the algorithm, we treat $\pi\left(K_{t+1} \mid \hat{\eta}_{t}\right)$ and $\pi\left(K_{t+1} \mid \tilde{q}_{t}\right)$ equivalently.

The state dynamics can be characterised by the transition probability function $\mathcal{P}\left(\tilde{q}_{t+1} \mid \tilde{q}_{t}, K_{t+1}\right)$. RL algorithms can be used to find the policy $\pi$, given a cost function ${ }^{1}$

\footnotetext{
${ }^{1}$ We will use cost instead of reward in this paper which is often used in control literature. Maximisation in RL setup will be minimisation instead.
} 
$C\left(\tilde{q}_{t}, K_{t+1}\right) \in \mathcal{C}$ that measures the goodness of a stateaction pair. In state estimation, it is often desired that the estimate error $\tilde{q}_{t}$ converges exponentially to a finite bound in mean square. As such, the cost function is selected as $C\left(\tilde{q}_{t}, K_{t+1}\right)=\mathbb{E}_{P\left(\cdot \mid \tilde{q}_{t}, K_{t+1}\right)}\left[\left\|\tilde{q}_{t+1}\right\|^{2}\right]$, and the return is the sum of discounted cost $\sum_{\tau=t}^{\infty} \gamma^{\tau-t} C\left(\tilde{q}_{t}, K_{t+1}\right)$ with the discount factor $\gamma \in[0,1)$.

Definition 1: [23] The estimate error $\tilde{q}_{t}$ in the MDP (7) is said to be exponentially bounded in mean square if $\exists \eta>0$ and $0<\varphi<1$, such that

$$
\mathbb{E}\left[\left\|\tilde{q}_{t}\right\|^{2}\right] \leq \eta \mathbb{E}\left[\left\|\tilde{q}_{0}\right\|^{2}\right] \varphi^{t}+p,
$$

holds at all the time instants $t \geq 0$, where $p$ is a positive constant number.

In this paper, our aim is to learn the estimator gain $K_{t+1}=$ $\pi\left(\hat{\eta}_{t}^{\mathrm{n}}\right)$ in (3) as a policy using RL, as showed in Fig 1. We expect that the mean square of the estimate error of $\tilde{q}_{t}$ in (7) is guaranteed to converge exponentially to a positive bound. Different from the EKF where $K_{t+1}$ is computed using the linearisation approximation, in this paper $K_{t+1}$ is approximated by a deep neural network $\pi(\cdot)$.

\section{Estimation ERror Boundedness GuARANTEE}

In this section, we propose the main theorem on boundedness of the estimate error. Before proceeding further, some notations are to be defined. $\rho\left(\tilde{q}_{0}\right)$ denotes the distribution of the starting state estimate error $\tilde{q}_{0}$. The state distribution of state estimate error at a certain instant $t$ as $P\left(\tilde{q}_{t} \mid \rho, \pi, t\right)$ is defined in an iterative way: $P\left(\tilde{q}_{t+1}=s^{\prime} \mid \rho, \pi, t+1\right)=$ $\int_{S} P\left(\tilde{q}_{t}=s \mid \rho, \pi, t\right) P_{\pi}\left(s^{\prime} \mid s\right) d s$. The following assumption that will be used in proving the theorem is presented as follows:

Assumption 1: The Markov chain in (7) induced by a policy $\pi$ is ergodic with a unique distribution probability. That is, $\exists p_{\pi}(s)$, such that

$$
p_{\pi}(s)=\lim _{t \rightarrow \infty} P\left(\tilde{q}_{t}=s \mid \rho, \pi, t\right)
$$

Theorem 1: The error dynamics (7) is exponentially bounded in mean square if there exists a Lyapunov function $L\left(\tilde{q}_{t}\right): S \rightarrow R^{+}$and positive constants $\alpha_{1}, \alpha_{2}$ and $\delta$ such that

$$
\alpha_{1} \mathbb{E}_{\pi}\left[\left\|\tilde{q}_{t}\right\|^{2}\right]-\delta \leq L\left(\tilde{q}_{t}\right) \leq \alpha_{1} \mathbb{E}_{\pi}\left[\left\|\tilde{q}_{t}\right\|^{2}\right]
$$

and

$$
\begin{array}{r}
\lim _{N \rightarrow+\infty}\left[\ln \left(\mathbb{E}_{\tilde{q}_{t} \sim \mu_{N}}\left(\mathbb{E}_{\tilde{q}_{t+1} \sim P_{\pi}} L\left(\tilde{q}_{t+1}\right)\right)\right)\right. \\
\left.-\mathbb{E}_{\tilde{q}_{t} \sim \mu_{N}} \ln \left(L\left(\tilde{q}_{t}\right)\right)\right] \leq-\alpha_{2}
\end{array}
$$

where

$$
\mu_{N}(s) \triangleq \frac{1}{N} \sum_{t=0}^{N-1} P\left(\tilde{q}_{t}=s \mid \rho, \pi, t\right)
$$

Proof: We have

$$
\begin{aligned}
& \ln \left(\mathbb{E}_{\tilde{q}_{t} \sim \mu_{N}}\left(\mathbb{E}_{\tilde{q}_{t+1} \sim P_{\pi}} L\left(\tilde{q}_{t+1}\right)\right)\right. \\
= & \ln \left(\int_{S} \frac{1}{N} \sum_{t=0}^{N-1} P\left(\tilde{q}_{t}=s \mid \rho, \pi, t\right) \int_{S} P_{\pi}\left(s^{\prime} \mid s\right) L\left(s^{\prime}\right) d s^{\prime} d s\right) \\
= & \ln \left(\int_{S}\left(\int_{S} \frac{1}{N} \sum_{t=0}^{N-1} P\left(\tilde{q}_{t}=s \mid \rho, \pi, t\right) P_{\pi}\left(s^{\prime} \mid s\right) d s\right) L\left(s^{\prime}\right) d s^{\prime}\right) \\
= & \ln \left(\int_{S}\left(\frac{1}{N} \sum_{t=0}^{N-1} P\left(\tilde{q}_{t+1}=s^{\prime} \mid \rho, \pi, t+1\right)\right) L\left(s^{\prime}\right) d s^{\prime}\right) \\
= & \ln \left(\left(\frac{1}{N} \sum_{t=0}^{N-1} \int_{S} P\left(\tilde{q}_{t+1}=s^{\prime} \mid \rho, \pi, t+1\right)\right) L\left(s^{\prime}\right) d s^{\prime}\right) \\
\geq & \frac{1}{N} \sum_{t=0}^{N-1} \ln \left(\left(\int_{S} P\left(\tilde{q}_{t+1}=s^{\prime} \mid \rho, \pi, t+1\right)\right) L\left(s^{\prime}\right) d s^{\prime}\right)
\end{aligned}
$$

where the last inequality follows from the fact that $\ln (x)$ is a concave function on $R^{+}$. Similarly, noting that $-\ln (x)$ is a convex function we have

$$
\begin{aligned}
& -\mathbb{E}_{\tilde{q}_{t} \sim \mu_{N}} \ln L\left(\tilde{q}_{t}\right) \\
= & -\int_{S} \frac{1}{N} \sum_{t=0}^{N-1} P\left(\tilde{q}_{t}=s \mid \rho, \pi, t\right) \ln (L(s)) d s \\
= & \frac{1}{N} \sum_{t=0}^{N-1} \int_{S} P\left(\tilde{q}_{t}=s \mid \rho, \pi, t\right)(-\ln L(s)) d s \\
\geq & \frac{1}{N} \sum_{t=0}^{N-1}-\ln \left(\int_{S} P\left(\tilde{q}_{t}=s \mid \rho, \pi, t\right) L(s) d s\right)
\end{aligned}
$$

It follows from the above two inequalities that

$$
\begin{aligned}
& \ln \left(\mathbb{E}_{\tilde{q}_{t} \sim \mu_{N}}\left(\mathbb{E}_{\tilde{q}_{t+1} \sim P_{\pi}} L\left(\tilde{q}_{t+1}\right)\right)-\mathbb{E}_{\tilde{q}_{t} \sim \mu_{N}} \ln L\left(\tilde{q}_{t}\right)\right) \\
\geq & \frac{1}{N} \sum_{t=0}^{N-1} \ln \frac{\int_{S} P\left(\tilde{q}_{t+1}=s^{\prime} \mid \rho, \pi, t+1\right) L\left(s^{\prime}\right) d s^{\prime}}{\int_{S} P\left(\tilde{q}_{t}=s \mid \rho, \pi, t\right) L(s) d s} \\
\geq & \frac{1}{N} \sum_{t=0}^{N-1} \ln \frac{\mathbb{E}_{\tilde{q}_{t+1}} L\left(\tilde{q}_{t+1}\right)}{\mathbb{E}_{\tilde{q}_{t}} L\left(\tilde{q}_{t}\right)}
\end{aligned}
$$

Substituting the above into (11), we obtain

$$
\lim _{N \rightarrow+\infty} \frac{1}{N} \sum_{t=0}^{N-1} \ln \frac{\mathbb{E}_{\tilde{q}_{t+1}} L\left(\tilde{q}_{t+1}\right)}{\mathbb{E}_{\tilde{q}_{t}} L\left(\tilde{q}_{t}\right)} \leq-\alpha_{2}
$$

then

$$
\lim _{N \rightarrow+\infty} \frac{1}{N} \ln \frac{\mathbb{E}_{\tilde{q}_{N}} L\left(\tilde{q}_{N}\right)}{\mathbb{E}_{\tilde{q}_{0}} L\left(\tilde{q}_{0}\right)} \leq-\alpha_{2}
$$

It means that $\forall \epsilon>0, \exists N_{\epsilon}, \frac{1}{N} \ln \frac{\mathbb{E}_{\tilde{q}_{N}} L\left(\tilde{q}_{N}\right)}{\mathbb{E}_{\tilde{q}_{0}} L\left(\tilde{q}_{0}\right)}<-\alpha_{2}+\epsilon<0$ holds when $N>N_{\epsilon}$, namely

$$
\frac{\mathbb{E}_{\tilde{q}_{N}} L\left(\tilde{q}_{N}\right)}{\mathbb{E}_{\tilde{q}_{0}} L\left(\tilde{q}_{0}\right)} \leq \mathrm{e}^{N\left(-\alpha_{2}+\epsilon\right)}, \forall N>N_{\epsilon}
$$

So we get for sufficiently large $N>N_{\epsilon}$,

$$
\mathbb{E}_{\tilde{q}_{N} \sim P\left(\tilde{q}_{N} \mid \rho, \pi, N\right)} L\left(\tilde{q}_{N}\right) \leq \mathrm{e}^{N\left(-\alpha_{2}+\epsilon\right)} \mathbb{E}_{\tilde{q}_{0} \sim \rho\left(\tilde{q}_{0}\right)} L\left(\tilde{q}_{0}\right)
$$


By Equation (10) we have the following result

$$
\begin{aligned}
& \mathbb{E}_{\tilde{q}_{N} \sim P\left(\tilde{q}_{N} \mid \rho, \pi, N\right)} \mathbb{E}_{\pi}\left\|\tilde{q}_{N}\right\|^{2} \\
\leq & \mathrm{e}^{N\left(-\alpha_{2}+\epsilon\right)} \mathbb{E}_{\tilde{q}_{0} \sim \rho\left(\tilde{q}_{0}\right)} \mathbb{E}_{\pi}\left\|\tilde{q}_{0}\right\|^{2}+\frac{\delta}{\alpha_{1}}
\end{aligned}
$$

So far, it has been proved that the estimate error $\tilde{q}_{t}$ in (7) is exponentially bounded according to Definition 1.

\section{LyapunOV-BASED REINFORCEMENT LEARNING ORIENTATION ESTIMATION ALGORITHM}

In this section, we will combine Soft Actor-Critic (SAC) algorithm [17], one of the state-of-the-art RL algorithms, with the theoretical result in Section III to learn the gain policy $K_{t+1}$ for the state estimator (3).

Considering MDP in (7), the orientation estimation problem can be viewed as a RL problem in which the policy is sought after by minimising the expected accumulated cost, i.e., the value function of the MDP. Here a stochastic policy is chosen as $\pi\left(K_{t+1} \mid \tilde{q}_{t}\right) \sim \mathcal{N}\left(K_{t+1}\left(\tilde{q}_{t}\right), \sigma\right)$ from which the gain $K_{t+1}$ for a given state $\tilde{q}_{t}$ is sampled [24]. The corresponding Q-function (a.k.a, state-action value function) is given as:

$$
Q_{\pi}\left(\tilde{q}_{t}, K_{t+1}\right)=C_{t}\left(\tilde{q}_{t}, K_{t+1}\right)+\gamma \mathbb{E}\left[V_{\pi}\left(\tilde{q}_{t+1}\right)\right]
$$

To this end, $K_{t+1}$ can be learned by many existing RL algorithms.

Motivated by the works in [20], [25], [26], [27], we propose to incorporate the theoretical result in Theorem 1 to formulate a constrained optimisation problem, based on SAC [17]. First of all, a Lyapunov candidate needs to be selected at the first instance. In the context of RL, a Lyapunov candidate will be parametrised/selected as the Q-function [19], [28]. In this paper, we choose $L\left(\tilde{q}_{t}\right)$ in 10 as:

$$
\left.L_{(} \tilde{q}_{t}\right)=\mathbb{E}_{K_{t+1} \sim \pi}\left[L_{c}\left(\tilde{q}_{t}, K_{t+1}\right)\right]
$$

where $L_{c}\left(\tilde{q}_{t}, K_{t+1}\right)=Q\left(\tilde{q}_{t}, K_{t+1}\right)$. Then the constrained optimisation problem is:

$$
\begin{array}{ll}
\min _{\pi} & Q_{\pi}\left(\tilde{q}_{t}, K_{t+1}\right) \\
\text { s.t. } & 100 \text { and } 11) \\
& -\ln \left(\pi\left(K_{t+1} \mid \tilde{q}_{t}\right)\right) \geq \mathcal{H}_{t}
\end{array}
$$

where $Q_{\pi}\left(\tilde{q}_{t}, K_{t+1}\right)$ is defined in 21), the second constraint is the minimum entropy constraint used in the SAC to improve the exploration in the action space [29] where $\mathcal{H}_{t}$ is the desired bound.

Denote the parametrised actor and critic as $\pi_{\theta}\left(K_{t+1} \mid \tilde{q}_{t}\right)$ and $Q_{\phi}\left(\tilde{q}_{t}, K_{t+1}\right)$ respectively, where $\theta$ and $\phi$ are the parameters of the DNNs. To ensure the positiveness of a Lyapunov function, $L_{\phi}\left(\tilde{q}_{t}, K_{t+1}\right)$ is selected as the square of a deep neural network as $L_{\phi}\left(\tilde{q}_{t}, K_{t+1}\right)=f_{\phi}^{\top}\left(\tilde{q}_{t}, K_{t+1}\right) f_{\phi}\left(\tilde{q}_{t}, K_{t+1}\right)$, where $f$ is the vector output of a DNN with parameters $\phi$. On the other hand, the stochastic policy $\pi_{\theta}\left(K_{t+1} \mid \tilde{q}_{t}\right)$ is parametrised by a deep neural network $f_{\theta}$ that depends on the state $\tilde{q}_{t}$ and a Gaussian noise $\epsilon$.

Solving the above constrained optimisation problem is equivalent to minimising the following objective function:

$$
\begin{aligned}
J(\theta) & =\mathbb{E}_{\tilde{q}_{t}, a_{t}, \tilde{q}_{t+1}, c_{t} \sim D}\left[\alpha\left(\ln \left(\pi_{\theta}\left(f_{\theta}\left(\tilde{q}_{t}, \epsilon\right) \mid \tilde{q}_{t}\right)\right)+\mathcal{H}_{t}\right)\right. \\
& \left.+\lambda\left(\ln L_{\phi}\left(\tilde{q}_{t+1}, f_{\theta}\left(\tilde{q}_{t+1}, \epsilon\right)\right)-\ln L\left(\tilde{q}_{t}, a_{t}\right)+\alpha_{2}\right)\right]
\end{aligned}
$$

TABLE I

HYPERPARAMETERS OF THE PROPOSED ESTIMATOR

\begin{tabular}{lc}
\hline Hyperparameters & Value \\
\hline Time horizon & 1000 \\
SGD batch size & 256 \\
Actor learning rate & $1 \mathrm{e}-4$ \\
Critic learning rate & $3 \mathrm{e}-4$ \\
Lyapunov learning rate & $3 \mathrm{e}-4$ \\
Target entropy & $\mathrm{NAN}$ \\
Soft replacement $(\tau)$ & $5 \mathrm{e}-3$ \\
Discount $(\gamma)$ & 0.999 \\
$\alpha_{3}$ & 0.2 \\
Structure of $a_{\phi}$ & $(128,64,32)$ \\
Structure of $L_{\theta}$ & $(128,64,32)$ \\
\hline
\end{tabular}

where $\mathcal{D}$ is the replay memory of the training samples, $\alpha$ and $\lambda$ are Lagrange multipliers which control the relative importance of constraints in 23 .

In the actor-critic framework, the parameters of policy network are updated through stochastic gradient descent of (24). It can be proved that the policy can converge to an optimal one that ensures the orientation estimate error $\mathbb{E}\left[\left\|\tilde{q}_{t}\right\|^{2}\right]$ converges to a constant as $t \rightarrow \infty, \forall \tilde{q}_{t} \in S$. The proof is standard and omitted due to page limits. The readers can refer to Section IV-D in [25] for more details. Pseudo code of the proposed Lyapunov-based reinforcement learning orientation estimation (LRLOE) algorithm is shown in Algorithm 1 .

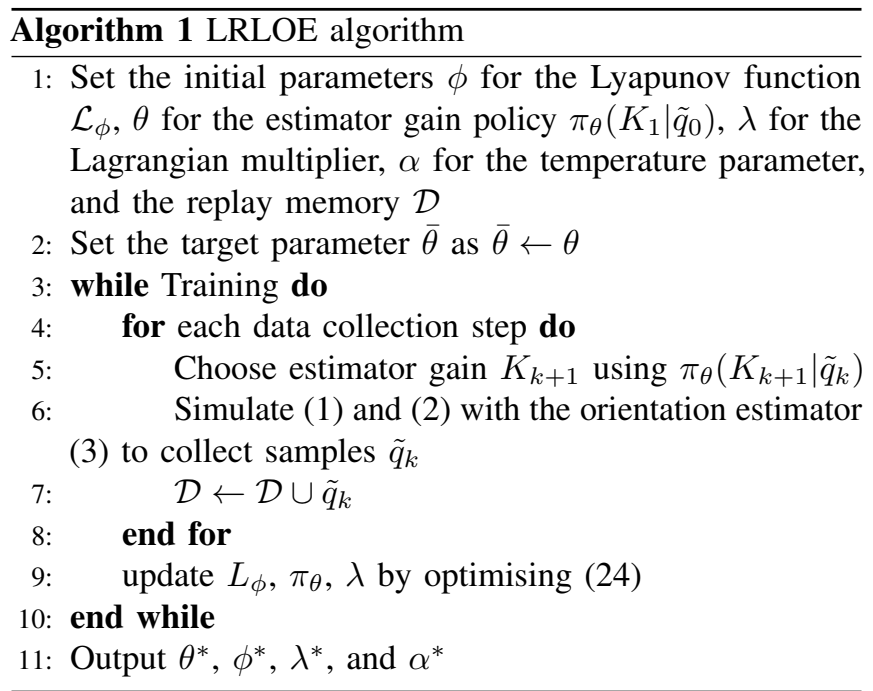

\section{EXPERIMENTAL RESUlts}

In this section, we train and inference on both simulated and real datasets. The estimation results has exhibited good performance, compared with three well-known oirentation estimation algorithms: EKF [5], UKF [10], and complementary filter [6].

\section{A. Results for simulated dataset}

The RL policy is trained on a relatively trivial profile (see figure. 2(a), then tested on three other independent profiles 


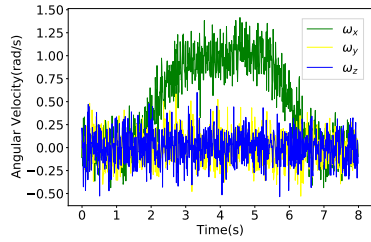

(a) Training profile

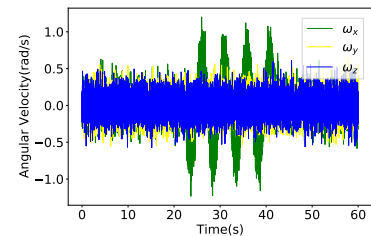

(c) Inference profile (medium)

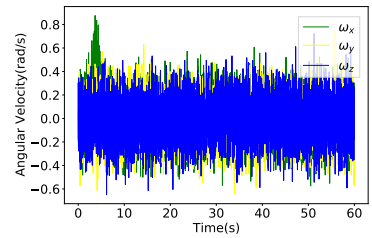

(b) Inference profile (simple)

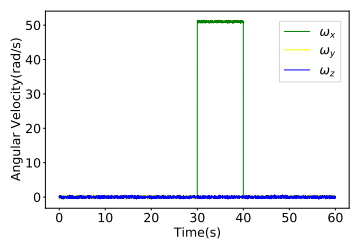

(d) Inference profile (complicated)
Fig. 2. The angular velocity profiles used for training and inference.

(see figure. 2(b), 2(c) and 2(d)]. The sampling rate is set to $100 \mathrm{~Hz}$ (consistent with real data in Section V-B). The sensor noise are sampled with the following distribution [3]:

$$
\begin{aligned}
e_{\omega, t} & \sim \mathcal{N}\left(0, \Sigma_{\omega}\right), & & \Sigma_{\omega}=0.0003, \\
e_{\mathrm{a}, t} & \sim \mathcal{N}\left(0, \Sigma_{\mathrm{a}}\right), & & \Sigma_{\mathrm{a}}=0.0005, \\
e_{\mathrm{m}, t} & \sim \mathcal{N}\left(0, \Sigma_{\mathrm{m}}\right), & & \Sigma_{\mathrm{m}}=0.0003
\end{aligned}
$$

During inference, the covariance of the measurement noise is increased as $\Sigma_{\omega}$ to 0.03 . The hyperparameters of Algorithm 1 are showed in Table I. The last $20 \%$ of training data is used for validation. We independently train 20 policies and select the one with the lowest validation error for inference. The initial state estimate is sampled from a normal distribution around the true initial orientation with standard deviation of 0.1 . The inference results of our proposed method are shown in Fig. 3, showing accurate estimation for all three different angular velocity profiles.

Furthermore, We compare our proposed DRL-based estimation method with the EKF [5], the UKF [10], and the complementary filter [6]. The root of mean square errors (RMSE) of the decoupled Euler parameters for pitch, $\phi$, roll, $\theta$ and heading, $\psi$ angles, corresponding to rotations around $\mathrm{x}, \mathrm{y}, \mathrm{z}$ axis respectively is chosen as the estimation performance and 200 Monte Carlo simulations are run for each algorithm. As shown in Fig. 4, the DRL-based estimation method achieves good performance as the EKF and exceeds the UKF and complementary filter under the inference profiles in Fig. 2(c).

The orientation estimation of the EKF, UKF and the complementary filter for a lage angular velocity are shown in Fig. 5. It can be found that the UKF yields a large estimate error under high noise level, and both the EKF and complementary filter perform poor as the estimate error accumulates in the long period of rapid rotation. In comparison, our proposed DRL-based estimation method has learnt error compensation from a large training dataset, thus showing more robust to different movement profiles.

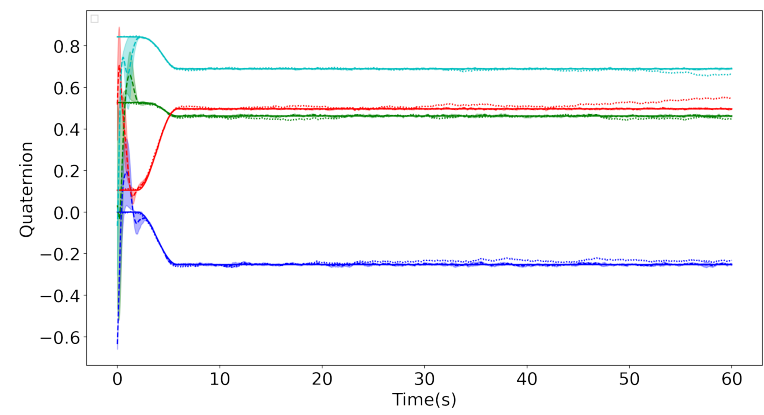

(a) Quaternion for Fig 2(b)

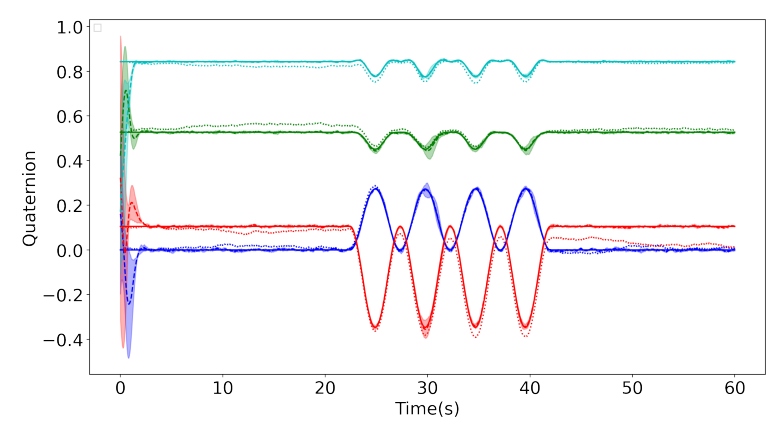

(b) Quaternion for Fig 2(c)

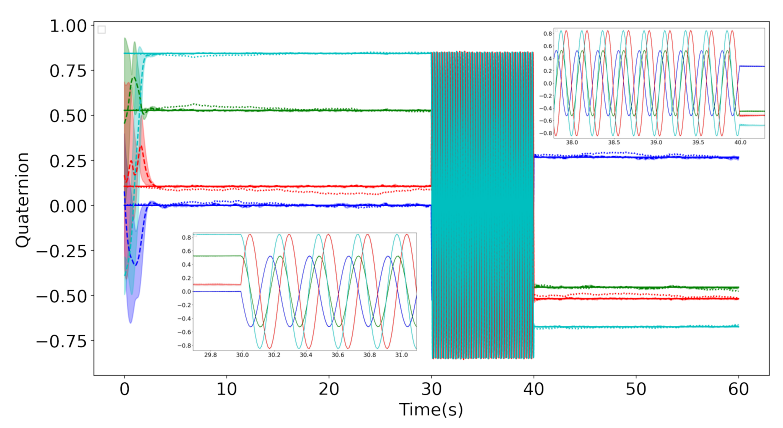

(c) Quaternion for Fig 2(d)

Fig. 3. Quaternion for different angular velocities in Fig.2(b) 2(c) and 2(d) The solid, dashed and dotted lines correspond to ground truth, measured and estimated quaternion respectively. The shaded areas correspond to the standard deviation over 20 independent runs. For the complicated profile, a zoomed estimation profile for high angular velocity is also showed in Fig. 3(c)

\section{B. Results for real dataset}

We apply our algorithm for real dataset from [3] (see Fig. 6). The data is collected from the Trivisio Colibri Wireless IMU [30] with a logging rate of $100 \mathrm{~Hz}$. The reference measurement of the orientation is provided as ground truth from a motion capture equipment [31] by tracking the optimal markers fixed to the sensor platform. The optical and IMU data has been time-synchronised and aligned beforehand.

The dataset is 100 seconds long and splited into training and inference dataset separately. The first half of the collected data is used for training and the rest for inference. In the training dataset, we randomly selected with a consecutive sequence of a length of 1000 samples as a training episode. We test in two scenarios (see Fig. 6): (1) inference including the training dataset from $t=0$ where the initial estimation $\hat{q}_{t=0 s}^{\text {nb }}$ 


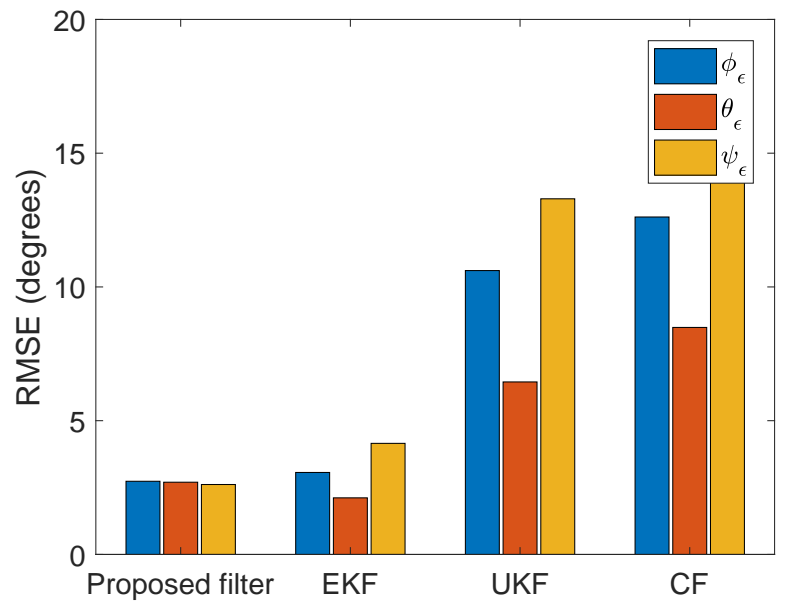

Fig. 4. The RMSE of the proposed filter, Extended Kalman filter (EKF), Unscented Kalman filter (UKF) and Complementary filter (CF) for the inference profiles in Fig. 2(c) 3(b) and 2(d)

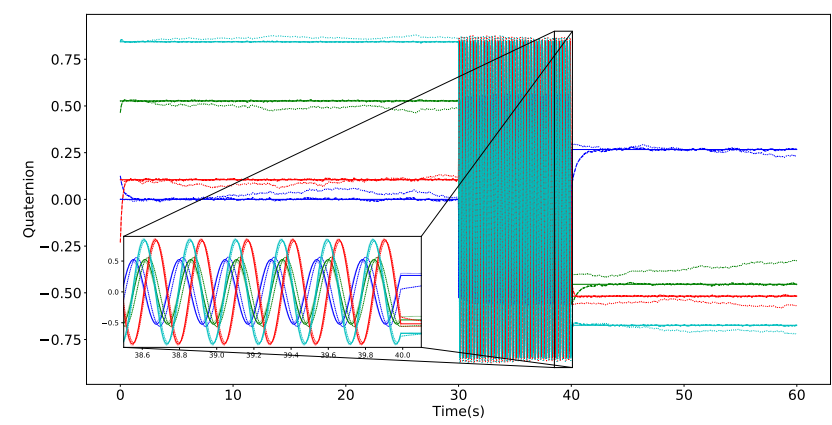

(a) EKF based quaternion estimation for Fig 2(d)

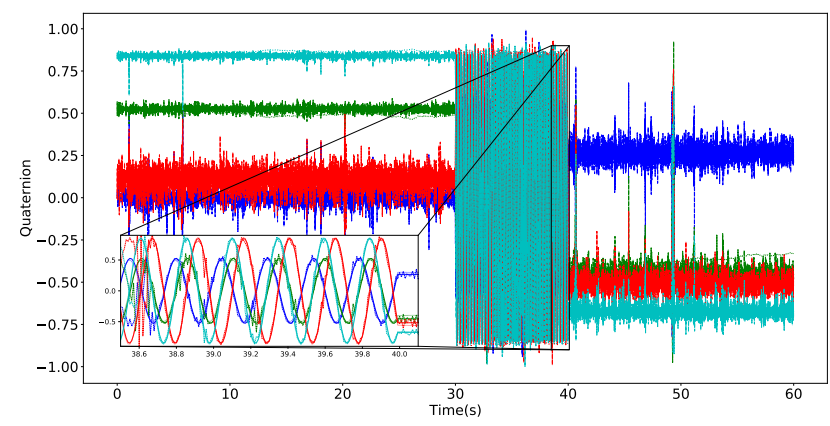

(b) UKF based quaternion estimation for Fig 2(d)

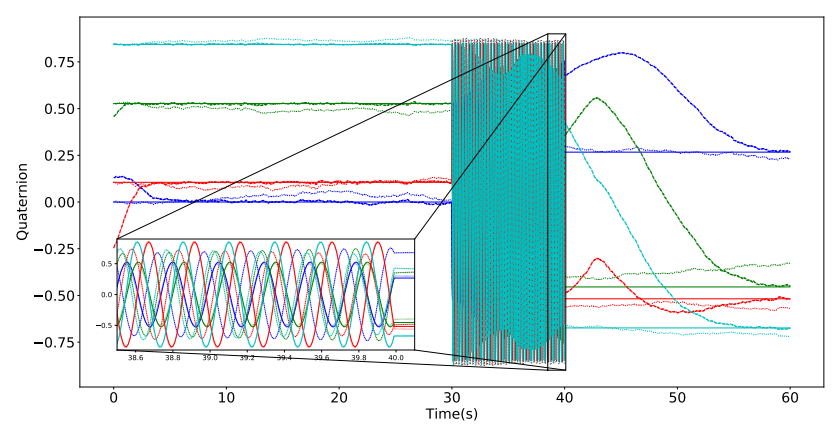

(c) CF based quaternion estimation in Fig 2(d)

Fig. 5. Comparison with other classic estimation algorithms for large angular velocities in Fig. 2(d)

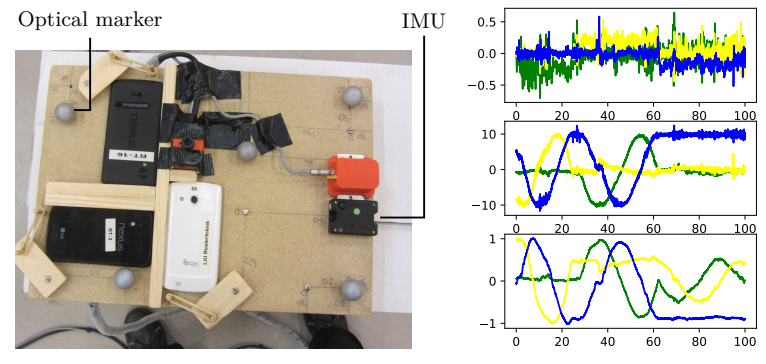

Fig. 6. Real dataset (adapted from Fig. 4.2 and 4.3 in [3]). Left: A snapshot of the platform for collecting real dataset Right: Measurements from an accelerometer ( $y_{\mathrm{a}, t}$, top), a gyroscope $\left(y_{\omega, t}\right.$, middle) and a magnetometer $\left(y_{\mathrm{m}, t}\right.$, bottom) for 100 seconds of data collected with the IMU shown in the left figure.).
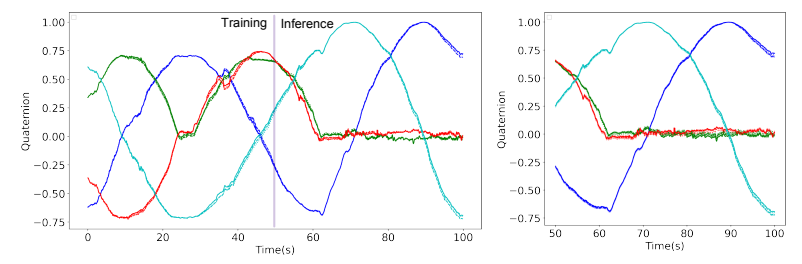

Fig. 7. Quaternion for real data. The solid, dashed and dotted lines correspond to ground truth, measured and estimated quaternion respectively. The left and right figures are corresponding for scenarios 1 and 2 respectively. In the right figure, the dashed line is obtained by averaging over 50 independent trails.

is simply the measurement; (2) inference without training dataset from $t=50 \mathrm{~s}$ where $\hat{q}_{t=50 \mathrm{~s}}^{\mathrm{nb}}$ is normally distributed around the true initial orientation with a standard deviation of 0.01. 50 independent trials are performed. The estimation results are showed in Fig. 7

TABLE II

RMSE OF THE ORIENTATION ESTIMATES

\begin{tabular}{lccc}
\hline RMSE & Yaw $\left[{ }^{\circ}\right]$ & ${\text { Pitch }\left[{ }^{\circ}\right]}$ & Roll $\left[{ }^{\circ}\right]$ \\
\hline Proposed algorithm & 1.9423 & 2.1048 & 0.8353 \\
Extended Kalman Filter & 2.0411 & 1.5272 & 1.2488 \\
Unscented Kalman Filter & 20.1370 & 20.3494 & 38.6775 \\
Complementary Filter & 1.2015 & 1.3892 & 0.8972 \\
\hline
\end{tabular}

In the second scenario, the second half of the dataset is also tested on the EKF, UKF and complementary filter methods. The estimation results are quantified and summarised in Table II Since the initial estimations and the gyroscope measurements are relatively accurate, results indicate that our proposed algorithm has achieved similar performance with the three state-of-art algorithms.

\section{CONCLUSION}

Orientation estimation using inertial sensors combined with magnetometer is well studied and many algorithms have been proposed. However, There hardly exist any algorithms with theoretical guarantees of estimation convergence. In this paper, we propose a reinforcement learning based orientation estimation method and prove that its estimate error converges to a positive scalar in mean square with a guarantee. The proposed method shows superior estimation performance 
compared with some well-known ones in terms of arbitrary estimation initialisation and adaptation to very large angular velocities.

\section{ACKNOWLEDGEMENT}

We thank Rick Staa at TU Delft for implementing the Algorithm 1 in TensorFlow [32]. We are grateful for the help and equipment provided by the UAS Technologies Lab, Artificial Intelligence and Integrated Computer Systems Division at the Department of Computer and Information Science, Linköping University, Sweden. We thank Gustaf Hendeby, Niklas Wahlström, Hanna Nyqvist and Manon Kok who collected the real data and allow us to use.

\section{REFERENCES}

[1] T. D. Barfoot, State estimation for robotics. Cambridge University Press, 2017.

[2] H. Zhou and H. Hu, "Human motion tracking for rehabilitation-a survey," Biomedical signal processing and control, vol. 3, no. 1, pp. 1-18, 2008.

[3] M. Kok, J. D. Hol, and T. B. Schön, "Using inertial sensors for position and orientation estimation," Foundations and Trends in Signal Processing, vol. 11, no. 1-2, pp. 1-153, 2017.

[4] A. M. Sabatini, "Quaternion-based extended kalman filter for determining orientation by inertial and magnetic sensing," IEEE transactions on Biomedical Engineering, vol. 53, no. 7, pp. 1346-1356, 2006.

[5] J. L. Marins, X. Yun, E. R. Bachmann, R. B. McGhee, and M. J. Zyda, "An extended kalman filter for quaternion-based orientation estimation using marg sensors," in Proceedings 2001 IEEE/RSJ International Conference on Intelligent Robots and Systems(IROS)., vol. 4. IEEE, 2001, pp. 2003-2011.

[6] R. G. Valenti, I. Dryanovski, and J. Xiao, "Keeping a good attitude: A quaternion-based orientation filter for imus and margs," Sensors, vol. 15 , no. 8, pp. 19302-19330, 2015.

[7] S. Wang, H. Wen, R. Clark, and N. Trigoni, "Keyframe based largescale indoor localisation using geomagnetic field and motion pattern," in 2016 IEEE/RSJ international conference on intelligent robots and systems (IROS). IEEE, 2016, pp. 1910-1917.

[8] R. Clark, S. Wang, H. Wen, A. Markham, and N. Trigoni, "Vinet: visual-inertial odometry as a sequence-to-sequence learning problem," in Proceedings of the Thirty-First AAAI Conference on Artificial Intelligence, 2017, pp. 3995-4001.

[9] T. Qin, P. Li, and S. Shen, "Vins-mono: A robust and versatile monocular visual-inertial state estimator," IEEE Transactions on Robotics, vol. 34, no. 4, pp. 1004-1020, 2018.

[10] E. Kraft, "A quaternion-based unscented kalman filter for orientation tracking," in Proceedings of the Sixth International Conference of Information Fusion, vol. 1, no. 1. IEEE Cairns, Queensland, Australia, 2003, pp. 47-54.

[11] S. O. Madgwick, A. J. Harrison, and R. Vaidyanathan, "Estimation of imu and marg orientation using a gradient descent algorithm," in 2011 IEEE international conference on rehabilitation robotics. IEEE, 2011, pp. 1-7.

[12] M. Kok, J. Hol, and T. Schön, "An optimization-based approach to human body motion capture using inertial sensors," in 19th World Congress of the International Federation of Automatic Control (IFAC), Cape Town, South Africa, August 24-29, 2014. International Federation of Automatic Control, 2014, pp. 79-85.

[13] M. Brossard, S. Bonnabel, and A. Barrau, "Denoising imu gyroscopes with deep learning for open-loop attitude estimation," arXiv preprint arXiv:2002.10718, 2020.

[14] J. Morimoto and K. Doya, "Reinforcement learning state estimator," Neural Computation, vol. 19, no. 3, pp. 730-756, 2007.

[15] T. P. Lillicrap, J. J. Hunt, A. Pritzel, N. Heess, T. Erez, Y. Tassa, D. Silver, and D. Wierstra, "Continuous control with deep reinforcement learning," arXiv preprint arXiv:1509.02971, 2015.

[16] J. Schulman, F. Wolski, P. Dhariwal, A. Radford, and O. Klimov, "Proximal policy optimization algorithms," arXiv preprint arXiv:1707.06347, 2017.
[17] T. Haarnoja, A. Zhou, P. Abbeel, and S. Levine, "Soft actor-critic: Offpolicy maximum entropy deep reinforcement learning with a stochastic actor," arXiv preprint arXiv:1801.01290, 2018.

[18] J.-J. E. Slotine, W. Li, et al., Applied nonlinear control. Prentice hall Englewood Cliffs, NJ, 1991, vol. 199, no. 1.

[19] V. Petridis and S. Petridis, "Construction of neural network based lyapunov functions," in The 2006 IEEE International Joint Conference on Neural Network Proceedings. IEEE, 2006, pp. 5059-5065.

[20] M. Han, L. Zhang, J. Wang, and W. Pan, "Actor-critic reinforcement learning for control with stability guarantee," IEEE Robotics and Automation Letters (RA-L \& IROS), accepted and in press, 2020. [Online]. Available: arXiv:2004.14288

[21] J. L. Crassidis, F. L. Markley, and Y. Cheng, "Survey of nonlinear attitude estimation methods," Journal of guidance, control, and dynamics, vol. 30, no. 1, pp. 12-28, 2007.

[22] J. Sola, "Quaternion kinematics for the error-state kf," Laboratoire dAnalyse et dArchitecture des Systemes-Centre national de la recherche scientifique (LAAS-CNRS), Toulouse, France, Tech. Rep, 2012.

[23] K. Reif, S. Gunther, E. Yaz, and R. Unbehauen, "Stochastic stability of the discrete-time extended kalman filter," IEEE Transactions on Automatic Control, vol. 44, no. 4, pp. 714-728, 1999.

[24] R. S. Sutton and A. G. Barto, Reinforcement Learning: An Introductions, 2nd ed. The MIT Press, 2018.

[25] L. Hu, C. Wu, and W. Pan, "Lyapunov-based reinforcement learning state estimator," arXiv preprint arXiv:2010.13529, 2020.

[26] Q. Zhang, W. Pan, and V. Reppa, "Model-reference reinforcement learning control of autonomous surface vehicles," in 2020 59th IEEE Conference on Decision and Control (CDC). IEEE, 2020, pp. 52915296.

[27] - , "Model-reference reinforcement learning for collision-free tracking control of autonomous surface vehicles," arXiv preprint arXiv:2008.07240, 2020.

[28] T. J. Perkins and A. G. Barto, "Lyapunov design for safe reinforcement learning," Journal of Machine Learning Research, vol. 3, no. Dec, pp. 803-832, 2002.

[29] T. Haarnoja, A. Zhou, P. Abbeel, and S. Levine, "Soft actor-critic: Offpolicy maximum entropy deep reinforcement learning with a stochastic actor," in Proceedings of the 35th International Conference on Machine Learning (ICML 2018), vol. 80, Stockholmsmässan, Stockholm Sweden, Jul. 2018, pp. 1861-1870.

[30] "Trivisio prototyping gmbh," http://www.trivisio.com, 2016

[31] "Vicon," url:http://www.vicon.com, Accessed on August 5., 2016.

[32] M. Abadi, P. Barham, J. Chen, Z. Chen, A. Davis, J. Dean, M. Devin, S. Ghemawat, G. Irving, M. Isard, et al., "Tensorflow: A system for large-scale machine learning," in 12th $\{$ USENIX $\}$ symposium on operating systems design and implementation $(\{O S D I\}$ 16), 2016, pp. 265-283. 